\title{
Induced star formation in the W40 region
}

\author{
Lev Pirogov*t \\ Institute of Applied Physics Russian Academy of Sciences, Russia \\ Lobachevsky State University of Nizhni Novgorod \\ E-mail: pirogoveappl.sci-nnov.ru
}

\begin{abstract}
A dense core associated with the W40 H II region has been observed in molecular lines and in continuum. The core consists of a clumpy dust ring and an extended molecular emission region. There are sharp differences in morphologies of dust and molecular gas. The ring is probably formed by the "collect \& collapse" process due to an expansion of neighbouring compact $\mathrm{H}$ II region into surrounding medium of fairly uniform and high density $\left(\sim 10^{5} \mathrm{~cm}^{-3}\right)$. There is a strong chemical differentiation in the ring. The $\mathrm{NH}_{3}, \mathrm{~N}_{2} \mathrm{H}^{+}$and $\mathrm{H}^{13} \mathrm{CO}^{+}$abundances are enhanced in the western clumps while the CS abundance is enhanced in the east. The eastern clumps which are closer to the main $\mathrm{H}$ II region are more massive and turbulent, show indications of infall and probably are more evolved than the western ones. An interaction between ionized gas and neutral material which is taking place in their vicinity triggers a new phase of star formation.
\end{abstract}

Frontier Research in Astrophysics

26 - 31 May, 2014

Mondello (Palermo), Italy

* Speaker.

${ }^{\dagger}$ A footnote may follow. 


\section{Introduction}

Early stages of star formation are still not clear despite an increasing number of observational data. This is especially true for the case of massive stars. High-mass star-forming (HMSF) regions are more rare, more distant than low-mass ones. Massive stars spend a significant time inside their parent cloud cores. During their evolution they affect surrounding cloud due to stellar winds, massive outflows, strong UV radiation and expansion of $\mathrm{H}$ II regions. These strong impacts change physical conditions and chemical composition of the parent cloud and may trigger the formation of a new generation of stars.

One of the nearby HMSF regions, W40, also known as Sharpless 64 [1], contains a large blister-type H II region that lies at the front edge of extended molecular cloud (TGU 279-P7 [2]) in the Aquila Rift complex. The H II region contains an embedded cluster of young stars including OB stars [3]. The distance to the cluster is $\sim 500 \mathrm{pc}$ [3] which puts W40 among the nearest HMSF regions. The associated W40 molecular cloud core is located to the west from the H II region. While expanding into the core the $\mathrm{H}$ II region compresses neutral material and probably triggers a new phase of star formation. In order to study this process in details we performed molecular multiline and dust continuum observations of W40 at millimeter wavelengths with IRAM-30, Onsala-20, Effelsberg-100 telescopes and also with GMRT array in decimeter wavelength continuum [5].

\section{Dust ring and ionized gas distribution}

A 1.2-mm dust continuum map of W40 [5] is shown in Fig. 1. The map reveals a ring-like structure consisting of dust clumps. Near-IR sources [3] and 3.6-cm compact VLA radio sources [7] are also shown. The IRS 1A South source (massive O9.5 star) is considered to be the driving source of the main H II region [3]. The IRS 5 infrared source which is a B1 star is the probable source of the neighbouring compact H II region [3, 8]. A part of the main $\mathrm{H}$ II region and the compact region centered at IRS 5 can be seen on the $1280 \mathrm{MHz}$ map (Fig. 1, left panel).

The dust ring consists of quite regularly spaced clumps (Fig. 1) which implies the "collect \& collapse" model of triggered star formation $[9,10]$. The ring is probably not being viewed face-on [5]. Class I and Class 0 sources associated with distinct clumps [6] indicate that star formation is going on there. The masses (Table 1$)$ and the sizes $(\sim 0.02-0.11 \mathrm{pc}[5])$ of the clumps are typical for low-mass cores. The dust temperatures of the clumps lie in the range $\sim 13-36 \mathrm{~K}$ [6]. Eastern clumps have higher masses and dust temperatures than the western ones.

\section{The "collect \& collapse" model parameters}

When an H II region expands into a medium consisting of neutral gas with uniform density a layer of enhanced density is forming between shock and ionization fronts. The layer splits into regular spaced fragments (clumps) by large-scale instabilities ( "collect \& collapse" model $[9,10]$ ). Such clumps can be sites of formation of the next generation of stars including massive ones. Such layers consisting of massive clumps have been found on a periphery of Galactic H II regions (e.g. [11]).

Using analytical expressions [10] we derived physical parameters of the fragments including the time when fragmentation in the layer occurs $\left(t_{f r a g}\right)$, the layer radius $\left(R_{\text {frag }}\right)$, the column density 


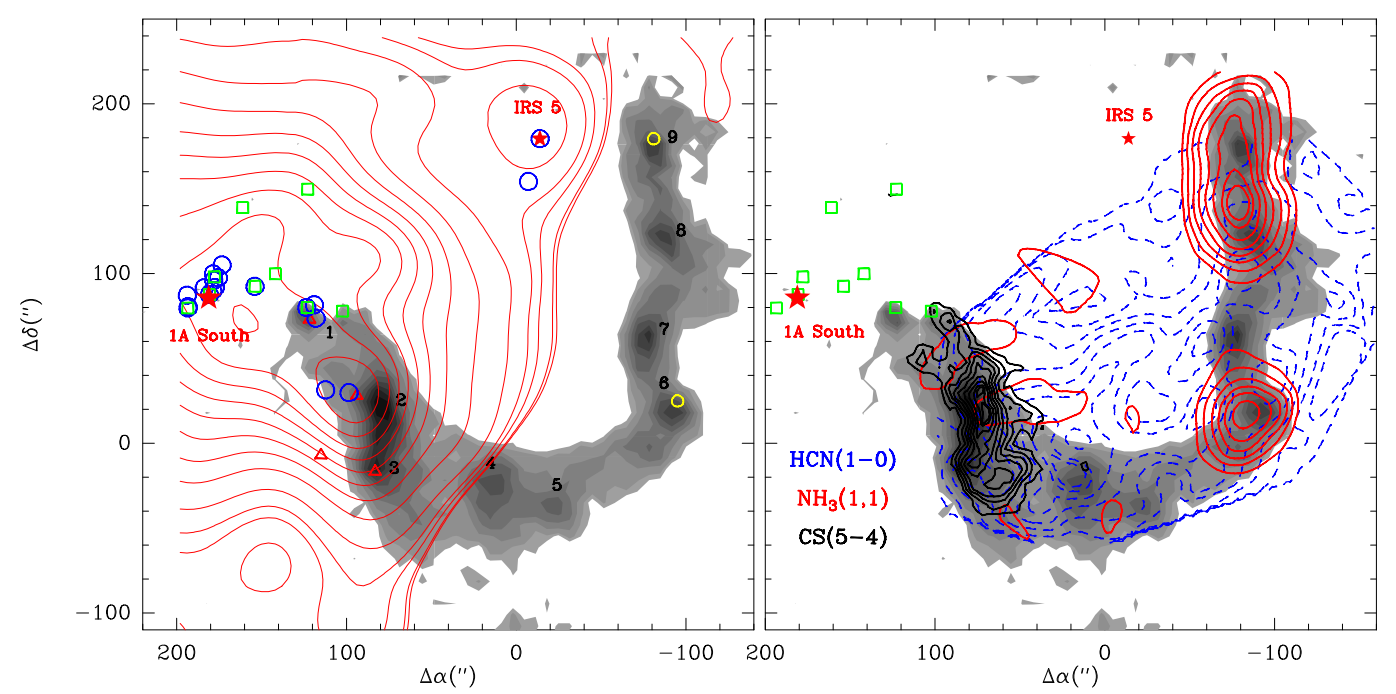

Figure 1: Dust, ionized and molecular gas in W40. Coordinates of the central position (the ${ }^{13} \mathrm{CO}$ peak [4]) are: R.A. $(\mathrm{J} 2000)=18^{h} 31^{m} 15.75^{s}$, Dec. $(\mathrm{J} 2000)=-02^{\circ} 06^{\prime} 49.3^{\prime \prime}$. The clumpy dust ring is shown in greyscale. Distinct clumps are denoted by numbers on the left panel. The ionized gas emission at $1280 \mathrm{MHz}$ is shown by red contours on the left panel. The $\mathrm{HCN}(1-0)$ (blue dashed contours), $\mathrm{NH}_{3}(1,1)$ (red contours) and $\mathrm{CS}(5-4)$ (black contours) are shown on the right panel. Near-IR sources [3] are shown as green squares. Compact VLA sources [7] are shown as blue circles (left panel). Class 0 and Class I sources [6] are marked by small yellow circles and red triangles, respectively (left panel). The driving sources of the main H II region (IRS 1A South) and the source of the neighbouring compact H II region (IRS 5) are indicated by stars.

of the layer $\left(N_{f r a g}\right)$, the mean mass of the fragments $\left(M_{\text {frag }}\right)$, and the initial separation between the fragments $\left(2 r_{\text {frag }}\right)$ (see [12] for details). These parameters depend on the luminosity of ionizing source as well as on the sound speed and the density of neutral gas. Kinetic temperature of neutral gas is assumed to be $\sim 10-15 \mathrm{~K}$. We took two luminosity values, the one close to the IRS 5 luminosity estimate from [8] and the other an order of magnitude higher. The model and the observed parameters are given in Table 1 . The corresponding dynamical age of the $\mathrm{H}$ II region $\left(t_{d y n}\right)$ is also calculated. The condition $t_{d y n} \gtrsim t_{\text {frag }}$ is satisfied in the calculations.

A comparison shows that the model with higher luminosity fits observations better. The small excess of the observed radius and column density of the layer over the model estimates could be connected to the evolution of these parameters during the time that has passed since the onset of fragmentation in the layer.

\section{Molecular line data. Modeling results}

Molecular integrated intensity maps overlayed over dust continuum are shown in Fig. 1 (right panel). The CS emission is strong towards the eastern branch of the ring while $\mathrm{NH}_{3}, \mathrm{~N}_{2} \mathrm{H}^{+}$and $\mathrm{H}^{13} \mathrm{CO}^{+}$are strong towards the western branch. The $\mathrm{HCN}, \mathrm{HCO}^{+}$and $\mathrm{CO}$ [4] emission is detected over extended part of the region and show practically no correlation with the dust.

Line profiles integrated over the eastern and western parts of the observed region are shown in Fig. 2. Towards the eastern part the observed lines can be roughly divided into two velocity ranges. The $\mathrm{HCN}(1-0)$ and $\mathrm{HCO}^{+}(1-0)$ lines are observed at $\sim 4.5-5 \mathrm{~km} \mathrm{~s}^{-1}$. The other lines 
Table 1: Observed and model clump parameters

\begin{tabular}{lccc}
\hline & Observed parameters & \multicolumn{2}{c}{ Model parameters } \\
\cline { 3 - 4 } & {$[5]$} & $L$ (photons s $\left.{ }^{-1}\right)=3 \cdot 10^{45}$ & $3 \cdot 10^{46}$ \\
\hline$n\left(\mathrm{~cm}^{-3}\right)$ & & $10^{5}$ & $1.5 \cdot 10^{5}$ \\
$R_{\text {frag }}(\mathrm{pc})$ & $\sim 0.3$ & 0.24 & 0.23 \\
$N_{\text {frag }}\left(10^{22} \mathrm{~cm}^{-2}\right)$ & $4-11$ & 2.4 & 3.6 \\
$M_{\text {frag }}\left(\mathrm{M}_{\odot}\right)$ & $2-6$ & 9.8 & 6.6 \\
$2 r_{\text {frag }}(\mathrm{pc})$ & $\sim 0.1-0.2$ & 0.27 & 0.18 \\
$t_{\text {dyn }}(\mathrm{Myr})$ & & 0.65 & 0.45 \\
$t_{\text {frag }}(\mathrm{Myr})$ & & 0.44 & 0.3 \\
\hline
\end{tabular}
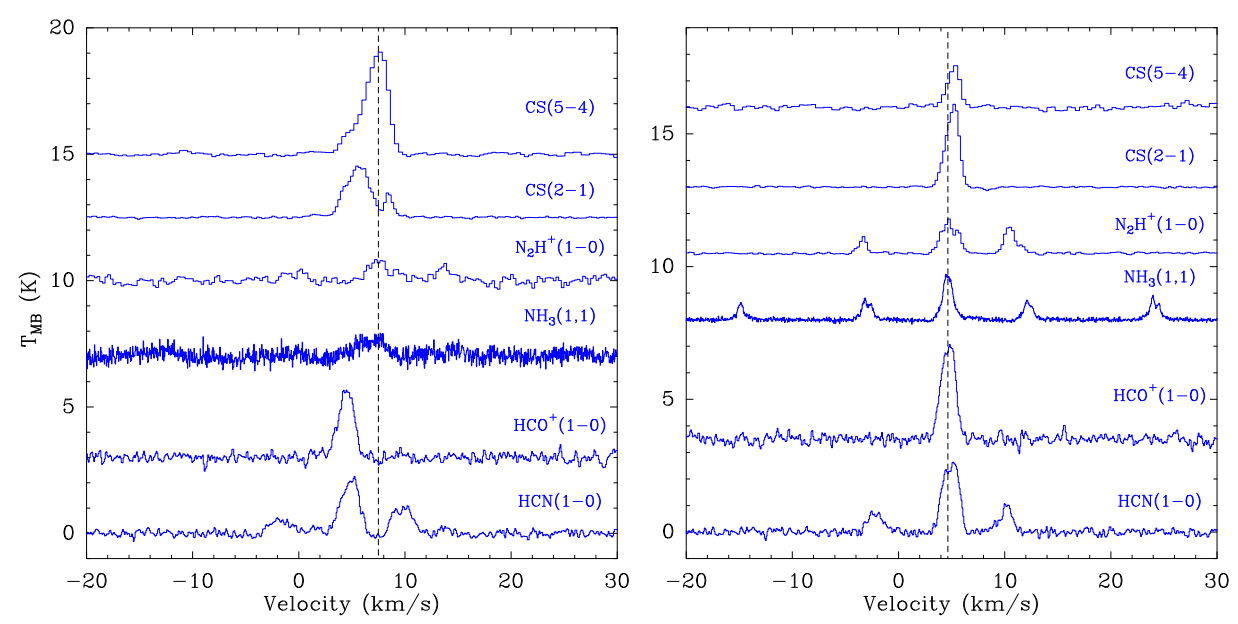

Figure 2: Molecular line spectra averaged over the eastern (left panel) $\left(\Delta \alpha=100^{\prime \prime} \ldots 40^{\prime \prime}, \Delta \delta=-50^{\prime \prime} \ldots 60^{\prime \prime}\right)$ and the western (right panel) $\left(\Delta \alpha=-50^{\prime \prime} \ldots-140^{\prime \prime}, \Delta \delta=-50^{\prime \prime} \ldots 60^{\prime \prime}\right)$ parts of the observed region. Dashed vertical lines correspond to $7.5 \mathrm{~km} \mathrm{~s}^{-1}$ (left panel) and to $4.65 \mathrm{~km} \mathrm{~s}^{-1}$ (right panel).

are observed at $\sim 7-8 \mathrm{~km} \mathrm{~s}^{-1}$. Line widths lie in the range: $\sim 1-2 \mathrm{~km} \mathrm{~s}^{-1}$ being more narrow in the western part. The gas in the eastern part is more turbulent.

Using both LTE approximation and non-LTE radiative transfer calculations the physical parameters of gas are derived [5]. Densities for selected positions lie in the range: $\sim(0.3-3)$. $10^{6} \mathrm{~cm}^{-3}$. Kinetic temperatures for two western clumps are $17 \mathrm{~K}$ and $21 \mathrm{~K}$, respectively, being close to dust temperatures. The western clumps are close to virial equilibrium.

Towards the eastern branch of the ring (the area including the dust clumps 2 and 3) the CS(21) profiles are self-absorbed and asymmetric. The velocity of the $\mathrm{CS}(2-1)$ absorption dip is close to the velocity of the CS(5-4) peak (Fig. 2, left panel) and also of the $\mathrm{C}^{34}(2-1)$ peak which is probably optically thin. This implies that the CS(2-1) line asymmetry is connected with systematic infall motions on the line of sight as predicted by the "inside-out" model [13]. We performed calculations of the CS excitation within the framework of a microturbulent multi-layer 1D sphericallysymmetric model with systematic infall motions and fit model profiles to the observed ones towards the $\left(80^{\prime \prime}, 40^{\prime \prime}\right)$ position. The model consists of a core and an envelope which effectively absorbs the CS(2-1) emission producing self-absorption dip on the CS(2-1) profile while the presence of infall motions makes the profile asymmetric. The observed and the model spectra are shown in Fig. 3. 


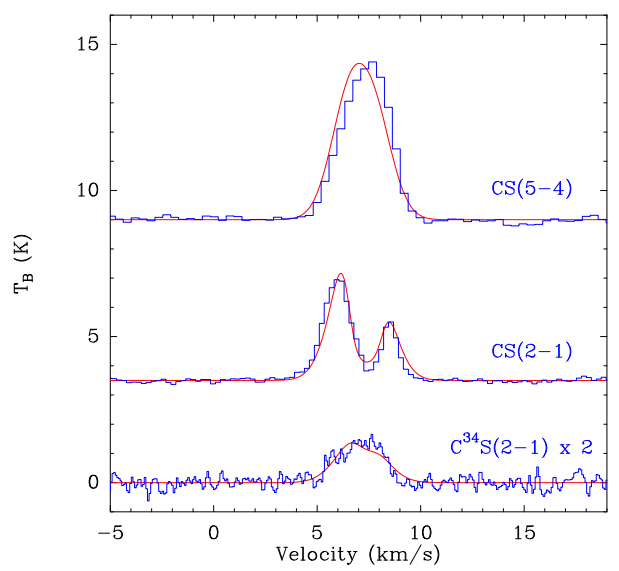

Figure 3: The $C S(5-4), C S(2-1)$ and $C^{34} S(2-1)$ line profiles towards the $\left(80^{\prime \prime}, 40^{\prime \prime}\right)$ position. The results of model calculations are shown by red smooth curves. The kinetic temperature is $40 \mathrm{~K}$, the infall velocity profile is $\propto 0.5 \mathrm{~km} \mathrm{~s}^{-1} r^{-0.1}$. The core density profile is $\propto 1.5 \cdot 10^{6} \mathrm{~cm}^{-3} r^{-2}$. The envelope density is $1.5 \cdot 10^{3} \mathrm{~cm}^{-3}$. More details are given in [5].

Table 2: Molecular and electron abundances

\begin{tabular}{rlllll}
\hline $\begin{array}{r}\Delta \alpha, \Delta \delta) \\
\left({ }^{\prime \prime},{ }^{\prime \prime}\right)\end{array}$ & $X\left(\mathrm{NH}_{3}\right)$ & $X\left(\mathrm{~N}_{2} \mathrm{H}^{+}\right)$ & $X(\mathrm{CS})$ & $X\left(\mathrm{H}^{13} \mathrm{CO}^{+}\right)$ & $X_{e}$ \\
\hline$(80,40)$ & $2.510^{-9}$ & $3.010^{-11}$ & $7.110^{-9}$ & & \\
$(80,0)$ & $1.510^{-9}$ & $3.810^{-11}$ & & $7.110^{-12}$ & \\
$(0,-40)$ & $2.610^{-9}$ & & $9.710^{-10}$ & & $1.710^{-7}$ \\
$(-100,20)$ & $9.310^{-9}$ & $5.610^{-10}$ & $1.110^{-9}$ & $3.110^{-11}$ & $3.110^{-7}$ \\
$(-80,140)$ & $1.510^{-8}$ & & $>410^{-10}$ & & \\
\hline
\end{tabular}

\section{Abundances}

Molecular and electron abundances for selected positions are given in Table 2. They are calculated as ratios of molecular column densities to the $\mathrm{H}_{2}$ column densities derived from the dust continuum data taking the gas-to-dust mass ratio equal to 100 . The abundances of nitrogenbearing molecules $\left(\mathrm{NH}_{3}\right.$ and $\left.\mathrm{N}_{2} \mathrm{H}^{+}\right)$and of $\mathrm{H}^{13} \mathrm{CO}^{+}$are enhanced towards the western positions $\left(-100^{\prime \prime}, 20^{\prime \prime}\right)$ and $\left(-80^{\prime \prime}, 140^{\prime \prime}\right)$ while $X(\mathrm{CS})$ is enhanced towards the eastern position $\left(80^{\prime \prime}, 40^{\prime \prime}\right)$ (dust clump 2). Electron abundances, $X(\mathrm{e})$, calculated using the $X\left(\mathrm{HCO}^{+}\right)$estimates are among the highest values for star-forming regions. Low values of $X\left(\mathrm{NH}_{3}\right)$ and $X\left(\mathrm{~N}_{2} \mathrm{H}^{+}\right)$in the central and in the eastern positions can be associated with enhanced ionized emission from the cluster stars and from IRS 5 which penetrates through inhomogeneous surrounding gas increasing electron abundances and destroying nitrogen-bearing molecules.

\section{Discussion}

The W40 cloud core consists of a clumpy dust ring formed by the "collect \& collapse" process and an extended dense core. The probable source of the ring could be IRS 5 if we take its luminosity 
higher than the value derived previously [8]. The observed ring structure represents a rare example of the "collect \& collapse" process at relatively small distances from the star in fairly homogeneous high-density environment $\left(\sim 10^{5} \mathrm{~cm}^{-3}\right)$. Note, that the shape of the high-resolution map of ionized gas in the vicinity of the eastern branch of the ring is similar to the molecular and dust maps [5] implying interaction of expanding ionization front from the main $\mathrm{H}$ II region with the eastern clumps of the ring.

The eastern branch of the ring differs in conditions from the other parts of the cloud. The eastern clumps 2 and 3 are more massive and turbulent and have higher dust temperatures. There is a strong chemical differentiation in the ring and indications of infall in the eastern branch of the ring. The eastern branch is more evolved than the western one probably because it is affected by the main driving source. We suggest that the area of the eastern clumps 2 and 3 could probably be a site of triggered formation of the stars with masses higher than one solar mass.

Acknowledgments I would like to thank D.K. Ojha, K.K. Mallick, M. Thomasson, Y.-F. Wu and I. Zinchenko for the help with observations and valuable discussions. I would like to thank referee Paolo Persi for the comment. The research made use of the SIMBAD database, operated by the CDS, Strasbourg, France. The research is supported in part by the Russian Foundation for Basic Research (grants 12-02-00861, 13-02-92697, 13-02-12220). The research is partly supported by the grant (the Agreement of August 27, 2013 N02.B.49.21.0003 between The Ministry of Education and Science of the Russian Federation and Lobachevsky State University of Nizhni Novgorod).

\section{References}

[1] Sharpless S.: 1959, ApJS, 4, 257.

[2] Dobashi K., Uehara H., Kandori R., et al.: 2005, PASJ, 57, SP1, 1.

[3] Shuping R.Y., Vacca W.D., Kassis M. \& Yu K.C.: 2012, AJ, 144, 116.

[4] Zhu L., Wu Y.-F. \& Wei Y.: 2006, Chin. J. A\&A, 6, 61.

[5] Pirogov L., Ojha D.K., Thomasson M., et al.: 2013, MNRAS 436, 3186.

[6] Maury A.J., André P., Menshchikov A., et al.: 2011, A\&A, 535, A77.

[7] Rodríguez L.F., Rodney S.A. \& Reipurth B.: 2010, AJ, 140, 968.

[8] Mallick K.K., Kumar M.S.N., Ojha D.K., et al.: 2013, ApJ, 779, 113.

[9] Elmegreen B.G. \& Lada C.J.: 1977, ApJ, 214, 725.

[10] Whitworth A.P., Bhattal A.S., Chapman S.J., et al.: 1994, MNRAS, 268, 291.

[11] Deharveng L., Schuller F., Anderson L.D., et al.: 2010, A\&A 523, A6.

[12] Pirogov L.E.: 2015, Astron.Rep., 59360.

[13] Evans II N.J.: 1999, ARA\&A, 37, 311.

\section{DISCUSSION}

ANA INÉS GOMEZ DE CASTRO: Is the separation between the clumps in the western area of the mapped region constant?

LEV PIROGOV: The separations between the clumps are close to each other except the clump 6 . This could be connected with local density inhomogenuity in the neutral gas in the shell. 